K. ŌSHIMA

KODAI MATH. J.

34 (2011), 291-300

\title{
HOMOTOPY GROUPS OF THE SPACE OF SELF MAPS OF THE PROJECTIVE 3-SPACE
}

\author{
KATSUMI ŌSHIMA
}

\begin{abstract}
We compute the homotopy groups of the space of self maps of the 3 dimensional projective space.
\end{abstract}

\section{Introduction}

For spaces $X$ and $Y$ with base points, we denote by $\operatorname{map}_{*}(X, Y)$ the space of maps from $X$ to $Y$ preserving base points. We take the trivial map 0 as the base point of $\operatorname{map}_{*}(X, Y)$. Homotopical properties of $\operatorname{map}_{*}(X, Y)$ have long been studied in algebraic topology. In the recent decade, several people have been interested in the case where $X$ is a Lie group and $X=Y[\mathbf{6}, \mathbf{7}, \mathbf{8}, 13,14,15,16]$. In the present note, we study the case $X=Y=\mathrm{SO}(3)=\mathbf{P}^{3}$ and we compute the homotopy groups $\pi_{n}\left(\operatorname{map}_{*}\left(\mathbf{P}^{3}, \mathbf{P}^{3}\right)\right)$ for $n \leq 20$, where $\mathbf{P}^{3}$ is the 3-dimensional projective space. As an application to our computations we know $\pi_{n}\left(\operatorname{aut}\left(\mathbf{P}^{3}\right)\right)$ for $n \leq 20$, where aut $\left(\mathbf{P}^{3}\right)$ is the space of self homotopy equivalences of $\mathbf{P}^{3}$. Results will be given in the section 2, and proofs will be given in sections 3,4 and 5.

I would like to thank Professor Ōshima for his help and support which made me go on with this work.

\section{Results}

The groups $\pi_{n}\left(\operatorname{map}_{*}\left(\mathbf{P}^{3}, \mathbf{P}^{3}\right)\right)$ for $n=0,1,3$ are well-known (cf. (3.1) below):

$$
\begin{aligned}
& \pi_{n}\left(\operatorname{map}_{*}\left(\mathbf{P}^{3}, \mathbf{P}^{3}\right)\right) \\
& \cong\left\{\begin{array}{lll}
\mathbf{Z} & n=0([\mathbf{1 2}, \text { Theorem IIa }] \text { or }[\mathbf{8}, \text { Proposition } 4.1]) \\
\left(\mathbf{Z}_{2}\right)^{2} & n=1([\mathbf{3},(9.1 .3)] \text { or }[\mathbf{6}, \text { Lemma } 7.3]) \\
\left(\mathbf{Z}_{4}\right)^{2} \oplus \mathbf{Z}_{3} & n=3([\mathbf{1 7}, \text { Corollary 5] or }[\mathbf{1 5}, \text { Lemma 2.1(6)] })
\end{array}\right.
\end{aligned}
$$

2000 Mathematics Subject Classification. primary 55Q05; secondary 55P10.

Key words and phrases. space of self maps; homotopy group; projective space.

Received September 28, 2010; revised December 16, 2010. 
Here $\mathbf{Z}_{k}$ denotes the cyclic group of order $k$ and $\left(\mathbf{Z}_{k}\right)^{m}$ is the direct sum of $m$ copies of $\mathbf{Z}_{k}$. We denote by $\mathbf{Z}_{k}\{x\}$ the cyclic group of order $k$ generated by $x$. We write $\left(\mathbf{Z}_{k}\right)^{m}\left\{x_{1}, \ldots, x_{m}\right\}=\mathbf{Z}_{k}\left\{x_{1}\right\} \oplus \cdots \oplus \mathbf{Z}_{k}\left\{x_{m}\right\}$.

We work in the category of spaces with base points, unless otherwise stated. The base point is denoted by $*$. We do not distinguish in notation between a map and its homotopy class. We denote the suspension functor by $E$, that is, $E^{n} X=X \wedge \mathrm{S}^{n}$ and $E^{n} f: E^{n} X \rightarrow E^{n} Y$ are the $n$-fold suspensions of a space $X$ and a map $f: X \rightarrow Y$, respectively. For spaces $X$ and $Y$, we denote the set of homotopy classes of maps from $X$ to $Y$ by $[X, Y]$, that is, $[X, Y]=$ $\pi_{0}\left(\operatorname{map}_{*}(X, Y)\right)$. We follow notations of $[\mathbf{1 8}]$ for elements of homotopy groups of spheres. Given a map $g: \mathrm{S}^{m} \rightarrow \mathrm{S}^{n}$ such that $2 g \simeq 0$, let $\bar{g}: \mathrm{S}^{m} \cup_{2 l_{m}} e^{m+1} \rightarrow \mathrm{S}^{n}$ denote an extension of $g$, that is, $\bar{g}=g$ on $\mathrm{S}^{m}$, where $\mathrm{S}^{m} \cup_{2 l_{m}} e^{m+1}$ is the mapping cone of $2 l_{m}$. We should be careful not to confuse Toda's elements $\bar{\varepsilon}_{n}$ (resp. $\bar{\mu}_{n}$ ) with extensions $\overline{\varepsilon_{n}}\left(\right.$ resp. $\overline{\mu_{n}}$ ) of Toda's elements $\varepsilon_{n}$ (resp. $\left.\mu_{n}\right)$. We set

$$
\Gamma_{n}=\left[\mathrm{S}^{n+1} \cup_{2 l_{n+1}} e^{n+2}, \mathrm{~S}^{3}\right] \quad(n \geq 0) .
$$

Notice that $\Gamma_{n}$ is a finite abelian group and $E^{n}\left(\mathrm{~S}^{1} \cup_{2 l_{1}} e^{2}\right)=\mathrm{S}^{n+1} \cup_{2 l_{n+1}} e^{n+2}$. We will prove the following assertion in $\S 3$.

Proposition 2.1. $\pi_{n}\left(\operatorname{map}_{*}\left(\mathbf{P}^{3}, \mathbf{P}^{3}\right)\right) \cong \Gamma_{n} \oplus \pi_{n+3}\left(\mathrm{~S}^{3}\right)$.

We refer $\pi_{n+3}\left(\mathrm{~S}^{3}\right)$ for $n \leq 20$ to $[\mathbf{1 8}, 9]$ (cf. Lemma 4.1(2) below).

In order to compute $\Gamma_{n}$, we use the following cofibre sequence

$$
\mathrm{S}^{n+2} \stackrel{-2 l_{n+2}}{\longleftarrow} \mathrm{S}^{n+2} \stackrel{q_{n}}{\longleftarrow} \mathrm{S}^{n+1} \cup_{2 l_{n+1}} e^{n+2} \stackrel{i_{n}}{\longleftarrow} \mathrm{S}^{n+1} \stackrel{2 l_{n+1}}{\longleftarrow} \mathrm{S}^{n+1} .
$$

Our main result is the following theorem which will be proved in $\S 4$.

THEOREM 2.2 .

\begin{tabular}{|c||c|c|c|c|c|c|}
\hline$n$ & 0 & 1 & 2 & 3 & 4 & 5 \\
\hline$\Gamma_{n}$ & 0 & $\mathbf{Z}_{2}$ & $\mathbf{Z}_{2}$ & $\mathbf{Z}_{4}$ & $\left(\mathbf{Z}_{2}\right)^{2}$ & $\left(\mathbf{Z}_{2}\right)^{2}$ \\
\hline generators & & $q_{1}$ & $q_{2}^{*} \eta_{3}$ & $\overline{\eta_{3}}$ & $q_{4}^{*} v^{\prime}, \eta_{3} \overline{\eta_{4}}$ & $q_{5}^{*}\left(v^{\prime} \eta_{6}\right), \eta_{3}^{2} \overline{\eta_{5}}$ \\
\hline relations & & & & $2 \overline{\eta_{3}}=q_{3}^{*} \eta_{3}^{2}$ & & \\
\hline
\end{tabular}

\begin{tabular}{|c|c|c|c|c|c|}
\hline 6 & 7 & 8 & 9 & 10 & 11 \\
\hline $\mathbf{Z}_{4}$ & $\mathbf{Z}_{2}$ & 0 & $\mathbf{Z}_{2}$ & $\mathbf{Z}_{2} \oplus \mathbf{Z}_{4}$ & $\left(\mathbf{Z}_{2}\right)^{2} \oplus \mathbf{Z}_{4}$ \\
\hline$v^{\prime} \overline{\eta_{6}}$ & $v^{\prime} \eta_{6} \overline{\eta_{7}}$ & & $q_{9}^{*} \varepsilon_{3}$ & $q_{10}^{*} \mu_{3}, \overline{\varepsilon_{3}}$ & $q_{11}^{*} \varepsilon^{\prime}, \varepsilon_{3} \overline{\eta_{11}}, \overline{\mu_{3}}$ \\
\hline $2\left(v^{\prime} \overline{\eta_{6}}\right)=q_{6}^{*}\left(v^{\prime} \eta_{6}^{2}\right)$ & & & & $2 \overline{\varepsilon_{3}}=q_{10}^{*}\left(\eta_{3} \varepsilon_{4}\right)$ & $2 \overline{\mu_{3}}=q_{11}^{*}\left(\eta_{3} \mu_{4}\right)$ \\
\hline
\end{tabular}




\begin{tabular}{|c|c|}
\hline 12 & 13 \\
\hline$\left(\mathbf{Z}_{2}\right)^{5}$ & $\left(\mathbf{Z}_{2}\right)^{3} \oplus \mathbf{Z}_{4}$ \\
\hline$q_{12}^{*} \mu^{\prime}, q_{12}^{*}\left(\varepsilon_{3} v_{11}\right), q_{12}^{*}\left(v^{\prime} \varepsilon_{6}\right), \varepsilon_{3} \eta_{11} \overline{\eta_{12}}, \mu_{3} \overline{\eta_{12}}$ & $q_{13}^{*}\left(v^{\prime} \mu_{6}\right), \mu_{3} \eta_{12} \overline{\eta_{13}}, \overline{\varepsilon_{3} v_{11}}, \varepsilon^{\prime} \overline{\eta_{13}}$ \\
\hline & $2\left(\varepsilon^{\prime} \overline{\eta_{13}}\right)=q_{13}^{*}\left(v^{\prime} \eta_{6} \varepsilon_{7}\right)$ \\
\hline
\end{tabular}

\begin{tabular}{|c|c|c|c|}
\hline 14 & 15 & 16 & 17 \\
\hline $\mathbf{Z}_{2} \oplus \mathbf{Z}_{4}$ & $\left(\mathbf{Z}_{2}\right)^{2}$ & $\left(\mathbf{Z}_{2}\right)^{2}$ & $\mathbf{Z}_{2} \oplus \mathbf{Z}_{4}$ \\
\hline$v^{\prime} \eta_{6} \overline{\varepsilon_{7}}, v^{\prime} \overline{\mu_{6}}$ & $q_{15}^{*}\left(\varepsilon_{3} v_{11}^{2}\right), v^{\prime} \eta_{6} \overline{\mu_{7}}$ & $q_{16}^{*} \bar{\varepsilon}_{3}, \varepsilon_{3} \overline{v_{11}^{2}}$ & $q_{17}^{*}\left(\mu_{3} \sigma_{12}\right), \overline{\bar{\varepsilon}_{3}}$ \\
\hline $2\left(v^{\prime} \overline{\mu_{6}}\right)=q_{14}^{*}\left(v^{\prime} \eta_{6} \mu_{7}\right)$ & & & $2 \overline{\bar{\varepsilon}}_{3}=q_{17}^{*}\left(\eta_{3} \bar{\varepsilon}_{4}\right)$ \\
\hline
\end{tabular}

\begin{tabular}{|c|c|}
\hline 18 & 19 \\
\hline$\left(\mathbf{Z}_{2}\right)^{3} \oplus \mathbf{Z}_{4}$ & $\left(\mathbf{Z}_{2}\right)^{4} \oplus \mathbf{Z}_{4}$ \\
\hline$q_{18}^{*} \bar{\varepsilon}^{\prime}, q_{18}^{*} \bar{\mu}_{3}, \bar{\varepsilon}_{3} \overline{\eta_{18}}, \overline{\mu_{3} \sigma_{12}}$ & $q_{19}^{*}\left(\mu^{\prime} \sigma_{14}\right), q_{19}^{*}\left(v^{\prime} \bar{\varepsilon}_{6}\right), \bar{\varepsilon}_{3} \eta_{18} \overline{\eta_{19}}, \mu_{3} \sigma_{12} \overline{\eta_{19}}, \overline{\mu_{3}}$ \\
\hline $2 \overline{\mu_{3} \sigma_{12}}=q_{18}^{*}\left(\eta_{3} \mu_{4} \sigma_{13}\right)$ & $2 \overline{\bar{\mu}}_{3}=q_{19}^{*}\left(\eta_{3} \bar{\mu}_{4}\right)$ \\
\hline
\end{tabular}

\begin{tabular}{|c|c|}
\hline 20 & 21 \\
\hline$\left(\mathbf{Z}_{2}\right)^{5}$ & $\left(\mathbf{Z}_{2}\right)^{2} \oplus \mathbf{Z}_{4}$ \\
\hline$q_{20}^{*} \bar{\mu}^{\prime}, q_{20}^{*}\left(v^{\prime} \mu_{6} \sigma_{15}\right), \mu_{3} \sigma_{12} \eta_{19} \overline{\eta_{20}}, v^{\prime} \overline{\bar{\varepsilon}}_{6}, \eta_{3} \overline{\bar{\mu}}_{4}$ & $q_{21}^{*}\left(v^{\prime} \bar{\mu}_{6}\right), \eta_{3}^{2} \overline{\bar{\mu}}_{5}, v^{\prime} \overline{\mu_{6} \sigma_{15}}$ \\
\hline & $2\left(v^{\prime} \overline{\mu_{6} \sigma_{15}}\right)=q_{21}^{*}\left(v^{\prime} \eta_{6} \mu_{7} \sigma_{16}\right)$ \\
\hline
\end{tabular}

Here we have used the following notations: $\overline{\eta_{n}}=E^{n-3} \overline{\eta_{3}}, \overline{\varepsilon_{n}}=E^{n-3} \overline{\varepsilon_{3}}, \overline{\mu_{n}}=$ $E^{n-3} \overline{\mu_{3}}, \overline{\bar{\varepsilon}_{n}}=E^{n-3} \overline{\bar{\varepsilon}}_{3}, \overline{\bar{\mu}}_{n}=E^{n-3} \overline{\bar{\mu}}_{3}$ and $\overline{\mu_{n} \sigma_{n+12}}=E^{n-3} \overline{\mu_{3} \sigma_{12}}$ for $n \geq 4$.

Rees [17, Corollary 5] determined $\Gamma_{3}$ by methods different with ours. By Proposition 2.1, Theorem 2.2 and $[\mathbf{1 8}, 9]$, we readily have

COROLlary 2.3 .

\begin{tabular}{|c||c|c|c|c|c|c|c|}
\hline$n$ & 0 & 1 & 2 & 3 & 4 & 5 & 6 \\
\hline$\pi_{n}\left(\operatorname{map}_{*}\left(\mathbf{P}^{3}, \mathbf{P}^{3}\right)\right)$ & $\mathbf{Z}$ & $\left(\mathbf{Z}_{2}\right)^{2}$ & $\left(\mathbf{Z}_{2}\right)^{2}$ & $\left(\mathbf{Z}_{4}\right)^{2} \oplus \mathbf{Z}_{3}$ & $\left(\mathbf{Z}_{2}\right)^{3}$ & $\left(\mathbf{Z}_{2}\right)^{3}$ & $\mathbf{Z}_{4} \oplus \mathbf{Z}_{3}$ \\
\hline
\end{tabular}

\begin{tabular}{|c|c|c|c|c|c|}
\hline 7 & 8 & 9 & 10 & 11 & 12 \\
\hline $\mathbf{Z}_{2} \oplus \mathbf{Z}_{3} \oplus \mathbf{Z}_{5}$ & $\mathbf{Z}_{2}$ & $\left(\mathbf{Z}_{2}\right)^{3}$ & $\left(\mathbf{Z}_{2}\right)^{2} \oplus\left(\mathbf{Z}_{4}\right)^{2} \oplus \mathbf{Z}_{3}$ & $\left(\mathbf{Z}_{2}\right)^{4} \oplus\left(\mathbf{Z}_{4}\right)^{2} \oplus \mathbf{Z}_{3} \oplus \mathbf{Z}_{7}$ & $\left(\mathbf{Z}_{2}\right)^{7}$ \\
\hline
\end{tabular}




\begin{tabular}{|c|c|c|c|}
\hline 13 & 14 & 15 & 16 \\
\hline$\left(\mathbf{Z}_{2}\right)^{4} \oplus \mathbf{Z}_{4} \oplus \mathbf{Z}_{3}$ & $\left(\mathbf{Z}_{2}\right)^{2} \oplus \mathbf{Z}_{4} \oplus \mathbf{Z}_{3} \oplus \mathbf{Z}_{5}$ & $\left(\mathbf{Z}_{2}\right)^{3} \oplus \mathbf{Z}_{3} \oplus \mathbf{Z}_{5}$ & $\left(\mathbf{Z}_{2}\right)^{4} \oplus \mathbf{Z}_{3}$ \\
\hline
\end{tabular}

\begin{tabular}{|c|c|c|c|}
\hline 17 & 18 & 19 & 20 \\
\hline$\left(\mathbf{Z}_{2}\right)^{3} \oplus\left(\mathbf{Z}_{4}\right)^{2} \oplus \mathbf{Z}_{3}$ & $\left(\mathbf{Z}_{2}\right)^{5} \oplus\left(\mathbf{Z}_{4}\right)^{2} \oplus \mathbf{Z}_{3}$ & $\left(\mathbf{Z}_{2}\right)^{5} \oplus\left(\mathbf{Z}_{4}\right)^{2} \oplus \mathbf{Z}_{3} \oplus \mathbf{Z}_{11}$ & $\left(\mathbf{Z}_{2}\right)^{7}$ \\
\hline
\end{tabular}

Let $\operatorname{aut}(X)$ denote the space of self homotopy equivalences of $X$ which are not necessarily preserving the base point, and aut ${ }_{*}(X)$ the space of based self homotopy equivalences. Then $\operatorname{aut}_{*}(X)$ is a submonoid of the monoid aut $(X)$ whose operation is the composition. By $[\mathbf{1 2}$, Theorem IIa, Theorem IIb] or $[\mathbf{1}$, Corollary 6], we have $\pi_{0}\left(\operatorname{aut}_{*}\left(\mathbf{P}^{3}\right)\right) \cong \pi_{0}\left(\operatorname{aut}\left(\mathbf{P}^{3}\right)\right) \cong \mathbf{Z}_{2}$. The following assertion will be proved in $\S 5$.

Proposition 2.4. If $n \geq 1$, then $\pi_{n}\left(\operatorname{aut}_{*}\left(\mathbf{P}^{3}\right)\right) \cong \Gamma_{n} \oplus \pi_{n+3}\left(\mathrm{~S}^{3}\right)$ and $\pi_{n}\left(\operatorname{aut}\left(\mathbf{P}^{3}\right)\right) \cong \Gamma_{n} \oplus \pi_{n+3}\left(\mathrm{~S}^{3}\right) \oplus \pi_{n}\left(\mathbf{P}^{3}\right)$.

\section{Proof of Proposition 2.1}

As is well-known, we have $\mathbf{P}^{3}=\mathrm{S}^{1} \cup_{2 l_{1}} e^{2} \cup e^{3}$ and

$$
\pi_{n}\left(\operatorname{map}_{*}\left(\mathbf{P}^{3}, \mathbf{P}^{3}\right)\right) \cong\left[\mathbf{P}^{3} \wedge \mathrm{S}^{n}, \mathbf{P}^{3}\right] .
$$

It follows from (2.1) that the assertion of Proposition 2.1 is true for $n=0,1$. By [4, (3.1)] (or [2]), we have $\mathbf{P}^{3} \wedge \mathrm{S}^{n} \simeq\left(\mathrm{S}^{n+1} \cup_{2 l_{n+1}} e^{n+2}\right) \vee \mathrm{S}^{n+3}$ for $n \geq 2$. Therefore

$$
\pi_{n}\left(\operatorname{map}_{*}\left(\mathbf{P}^{3}, \mathbf{P}^{3}\right)\right) \cong\left[\mathrm{S}^{n+1} \cup_{2 l_{n+1}} e^{n+2}, \mathbf{P}^{3}\right] \oplus \pi_{n+3}\left(\mathbf{P}^{3}\right) \text { if } n \geq 2 .
$$

The covering map $p: \mathrm{S}^{3} \rightarrow \mathbf{P}^{3}$ induces isomorphisms $\Gamma_{n} \cong\left[\mathrm{S}^{n+1} \cup_{2 l_{n+1}} e^{n+2}, \mathbf{P}^{3}\right]$ for $n \geq 1$ and $\pi_{n+3}\left(\mathbf{S}^{3}\right) \cong \pi_{n+3}\left(\mathbf{P}^{3}\right)$ for $n \geq 0$. Hence we have Proposition 2.1 for $n \geq 2$. This completes the proof of Proposition 2.1.

\section{Proof of Theorem 2.2} $k \neq 3$.

Let $\pi_{k}\left(\mathrm{~S}^{3} ; 2\right)$ be $\pi_{3}\left(\mathrm{~S}^{3}\right)$ if $k=3$ and the 2-primary subgroup of $\pi_{k}\left(\mathrm{~S}^{3}\right)$ if

By applying the cohomotopy functor $\left[, \mathrm{S}^{3}\right]$ to the cofibre sequence $(2.2)$, we have the following exact sequence of abelian groups.

$$
\pi_{n+2}\left(\mathrm{~S}^{3}\right) \stackrel{\left(-2 l_{n+2}\right)^{*}}{\longrightarrow} \pi_{n+2}\left(\mathrm{~S}^{3}\right) \stackrel{q_{n}^{*}}{\longrightarrow} \Gamma_{n} \stackrel{i_{n}^{*}}{\longrightarrow} \pi_{n+1}\left(\mathrm{~S}^{3}\right) \stackrel{\left(2 l_{n+1}\right)^{*}}{\longrightarrow} \pi_{n+1}\left(\mathrm{~S}^{3}\right) .
$$

Since $\left(2 l_{k}\right)^{*}: \pi_{k}\left(\mathbf{S}^{3}\right) \rightarrow \pi_{k}\left(\mathbf{S}^{3}\right)$ is the multiplication by $2,\left(-2 l_{k}\right)^{*}=\left(2 l_{k}\right)^{*}\left(-l_{k}\right)^{*}=$ $-\left(2 \imath_{k}\right)^{*}$ and $(-2) \pi_{k}\left(\mathrm{~S}^{3}\right)=2 \pi_{k}\left(\mathrm{~S}^{3}\right)$, it follows that the above exact sequence induces the following two exact sequences. 


$$
\begin{gathered}
\pi_{n+2}\left(\mathrm{~S}^{3}\right) \stackrel{2}{\rightarrow} \pi_{n+2}\left(\mathrm{~S}^{3}\right) \stackrel{q_{n}^{*}}{\rightarrow} \Gamma_{n} \stackrel{i_{n}^{*}}{\rightarrow}\left\{\beta \in \pi_{n+1}\left(\mathrm{~S}^{3}\right) \mid 2 \beta=0\right\} \rightarrow 0, \\
\pi_{n+2}\left(\mathrm{~S}^{3} ; 2\right) \stackrel{2}{\rightarrow} \pi_{n+2}\left(\mathrm{~S}^{3} ; 2\right) \stackrel{q_{n}^{*}}{\rightarrow} \Gamma_{n} \stackrel{i_{n}^{*}}{\rightarrow}\left\{\beta \in \pi_{n+1}\left(\mathrm{~S}^{3} ; 2\right) \mid 2 \beta=0\right\} \rightarrow 0 .
\end{gathered}
$$

We will use the following known results.

Lemma 4.1. (1) $([\mathbf{1 0},(2.3)])$. For every $n$, the suspension $E: \pi_{n}\left(\mathrm{~S}^{3}\right) \rightarrow$ $\pi_{n+1}\left(\mathrm{~S}^{4}\right)$ is an isomorphism onto a direct summand, that is, there is a homomorphism $\varphi_{n}: \pi_{n+1}\left(\mathrm{~S}^{4}\right) \rightarrow \pi_{n}\left(\mathrm{~S}^{3}\right)$ such that $\varphi_{n} \circ E$ is the identity.

(2) $([\mathbf{1 8}, \mathbf{9}])$. We have the following table:

\begin{tabular}{|c||c|c|c|c|c|c|c|c|c|c|c|}
\hline$n$ & 1,2 & 3 & 4 & 5 & 6 & 7 & 8 & 9,10 & 11 & 12 & 13 \\
\hline$\pi_{n}\left(\mathbf{S}^{3} ; 2\right)$ & 0 & $\mathbf{Z}$ & $\mathbf{Z}_{2}$ & $\mathbf{Z}_{2}$ & $\mathbf{Z}_{4}$ & $\mathbf{Z}_{2}$ & $\mathbf{Z}_{2}$ & 0 & $\mathbf{Z}_{2}$ & $\left(\mathbf{Z}_{2}\right)^{2}$ & $\mathbf{Z}_{2} \oplus \mathbf{Z}_{4}$ \\
\hline generator & & $\iota_{3}$ & $\eta_{3}$ & $\eta_{3}^{2}$ & $v^{\prime}$ & $v^{\prime} \eta_{6}$ & $v^{\prime} \eta_{6}^{2}$ & & $\varepsilon_{3}$ & $\mu_{3}, \eta_{3} \varepsilon_{4}$ & $\eta_{3} \mu_{4}, \varepsilon^{\prime}$ \\
\hline
\end{tabular}

\begin{tabular}{|c|c|c|c|c|c|c|}
\hline 14 & 15 & 16 & 17 & 18 & 19 & 20 \\
\hline$\left(\mathbf{Z}_{2}\right)^{2} \oplus \mathbf{Z}_{4}$ & $\left(\mathbf{Z}_{2}\right)^{2}$ & $\mathbf{Z}_{2}$ & $\mathbf{Z}_{2}$ & $\mathbf{Z}_{2}$ & $\left(\mathbf{Z}_{2}\right)^{2}$ & $\left(\mathbf{Z}_{2}\right)^{2} \oplus \mathbf{Z}_{4}$ \\
\hline$\varepsilon_{3} v_{11}, v^{\prime} \varepsilon_{6}, \mu^{\prime}$ & $v^{\prime} \mu_{6}, v^{\prime} \eta_{6} \varepsilon_{7}$ & $v^{\prime} \eta_{6} \mu_{7}$ & $\varepsilon_{3} v_{11}^{2}$ & $\bar{\varepsilon}_{3}$ & $\mu_{3} \sigma_{12}, \eta_{3} \bar{\varepsilon}_{4}$ & $\bar{\mu}_{3}, \eta_{3} \mu_{4} \sigma_{13}, \bar{\varepsilon}^{\prime}$ \\
\hline
\end{tabular}

\begin{tabular}{|c|c|c|}
\hline 21 & 22 & 23 \\
\hline$\left(\mathbf{Z}_{2}\right)^{2} \oplus \mathbf{Z}_{4}$ & $\mathbf{Z}_{2} \oplus \mathbf{Z}_{4}$ & $\left(\mathbf{Z}_{2}\right)^{2}$ \\
\hline$v^{\prime} \bar{\varepsilon}_{6}, \eta_{3} \bar{\mu}_{4}, \mu^{\prime} \sigma_{14}$ & $v^{\prime} \mu_{6} \sigma_{15}, \bar{\mu}^{\prime}$ & $v^{\prime} \bar{\mu}_{6}, v^{\prime} \eta_{6} \mu_{7} \sigma_{16}$ \\
\hline
\end{tabular}

For each $n$, we can write $\left\{\beta \in \pi_{n+1}\left(\mathbf{S}^{3} ; 2\right) \mid 2 \beta=0\right\}=\left(\mathbf{Z}_{2}\right)^{m}\left\{y_{1}, \ldots, y_{m}\right\}$ with $m \geq 0$ and $\pi_{n+2}\left(\mathbf{S}^{3} ; 2\right)=\mathbf{Z}_{2^{k_{1}}}\left\{x_{1}\right\} \oplus \cdots \oplus \mathbf{Z}_{2^{k_{l}}}\left\{x_{l}\right\}$ with $l \geq 0$ and $k_{i} \geq 1$ for every $i \leq l$. Hence (4.2) induces the following exact sequence:

$$
0 \rightarrow\left(\mathbf{Z}_{2}\right)^{l}\left\{q_{n}^{*}\left(x_{1}\right), \ldots, q_{n}^{*}\left(x_{l}\right)\right\} \stackrel{\subseteq}{\rightarrow} \Gamma_{n} \stackrel{i_{n}^{*}}{\rightarrow}\left(\mathbf{Z}_{2}\right)^{m}\left\{y_{1}, \ldots, y_{m}\right\} \rightarrow 0 .
$$

The following result can be proved easily. So we omit its proof.

Lemma 4.2. (1) If $2 \overline{y_{j}}=0$ for all $j$ in (4.3), then

$$
\Gamma_{n}=\left(\mathbf{Z}_{2}\right)^{l+m}\left\{q_{n}^{*}\left(x_{1}\right), \ldots, q_{n}^{*}\left(x_{l}\right), \overline{y_{1}}, \ldots, \overline{y_{m}}\right\} .
$$

(2) If $2 \overline{y_{j}}=0$ for all $j<m$ and $2 \overline{y_{m}}=q_{n}^{*}\left(x_{l}\right)$ in (4.3), then

$$
\Gamma_{n}=\left(\mathbf{Z}_{2}\right)^{l+m-2}\left\{q_{n}^{*}\left(x_{1}\right), \ldots, q_{n}^{*}\left(x_{l-1}\right), \overline{y_{1}}, \ldots, \overline{y_{m-1}}\right\} \oplus \mathbf{Z}_{4}\left\{\overline{y_{m}}\right\} .
$$

In order to determine the group extension of (4.3), we will compute $2 \overline{y_{j}}(1 \leq j \leq m)$ by using the following two lemmas. 
LeMma 4.3. (1) ([18]). $\pi_{n+4}\left(\mathrm{~S}^{n} ; 2\right)=0$ for $n \geq 6, \pi_{n+5}\left(\mathrm{~S}^{n} ; 2\right)=0$ for $n \geq 7$, $\pi_{n+6}\left(\mathbf{S}^{n} ; 2\right)=\mathbf{Z}_{2}\left\{v_{n}^{2}\right\} \quad$ for $\quad n \geq 5, \quad 2 v^{\prime}=\eta_{3}^{3}, \quad 4 v_{5}=\eta_{5}^{3}, \quad \eta_{10} \sigma_{11}=\sigma_{10} \eta_{17}$, $\eta_{3} \varepsilon_{4}=\varepsilon_{3} \eta_{11}, 2 \varepsilon^{\prime}=\eta_{3}^{2} \varepsilon_{5}, 2 \mu^{\prime}=\eta_{3}^{2} \mu_{5}, v^{\prime} \varepsilon_{6}=\varepsilon^{\prime} \eta_{13}, \eta_{3} \bar{\varepsilon}_{4}=\bar{\varepsilon}_{3} \eta_{18}=\varepsilon_{3}^{2}=\varepsilon_{3} \bar{v}_{11}$, $2 \bar{\varepsilon}^{\prime}=\eta_{3}^{2} \bar{\varepsilon}_{5}, 2 \bar{\mu}^{\prime}=\eta_{3}^{2} \bar{\mu}_{5}$.

(2) ([11]). $\eta_{3} \mu_{4}=\mu_{3} \eta_{12}, v^{\prime} \mu_{6}=\mu^{\prime} \eta_{14}, v^{\prime} \bar{\varepsilon}_{6}=\bar{\varepsilon}_{3} v_{18}, \eta_{3} \bar{\mu}_{4}=\bar{\mu}_{3} \eta_{20}$.

Proof. By Propositions 5.8 and 5.9, (5.3), (5.5), (7.5), (7.7), (7.12), Lemmas $6.4,6.6,12.3,12.4$ and 12.10 of $[\mathbf{1 8}]$, we have (1). We have (2) from Proposition $(2.2)(2),(4)$ and Proposition $(2.17)(4),(10)$ of $[11]$ which were proved by standard methods of $[\mathbf{1 8}]$.

LEMmA 4.4. If $\beta \in \pi_{n+1}\left(\mathrm{~S}^{3} ; 2\right)$ is of order 2, then every $\bar{\beta} \in\left[\mathrm{S}^{n+1} \cup_{2 l_{n+1}}\right.$ $\left.e^{n+2}, \mathrm{~S}^{3}\right]$ satisfies $2 \bar{\beta}=q_{n}^{*}\left(\beta \circ \eta_{n+1}\right)$.

Proof. Take $x \in\left\{2 \imath_{3}, \beta, 2 \imath_{n+1}\right\}_{0}$ arbitrarily, where $\left\{\gamma, E^{k} \delta, E^{k} \varepsilon\right\}_{k}$ is the Toda bracket $[\mathbf{1 8}]$. Then $\left\{2 \imath_{3}, \beta, 2 \imath_{n+1}\right\}_{0}=x+2 \pi_{n+2}\left(\mathrm{~S}^{3}\right)$ and

$$
\begin{aligned}
2 \bar{\beta} & =2 \imath_{3} \circ \bar{\beta} \in\left\{2 \imath_{3}, \beta, 2 \imath_{n+1}\right\}_{0} \circ q_{n} \quad(\text { by }[\mathbf{1 8}, \text { Proposition 1.9]) } \\
& =\left\{q_{n}^{*}(x)\right\} \quad(\text { by }(4.1)),
\end{aligned}
$$

that is, $2 \bar{\beta}=q_{n}^{*}(x)$. We have

$$
\begin{aligned}
E x & \in E\left\{2 \imath_{3}, \beta, 2 \imath_{n+1}\right\}_{0} \subset-\left\{2 \imath_{4}, E \beta, 2 \imath_{n+2}\right\}_{1} \quad(\text { by }[\mathbf{1 8}, \text { Proposition 1.3]) } \\
& =E\left(\beta \eta_{n+1}\right)+2 \pi_{n+3}\left(\mathrm{~S}^{4}\right) \quad(\text { by }[\mathbf{1 8}, \text { Corollary } 3.7]) .
\end{aligned}
$$

Hence there exists $y \in \pi_{n+3}\left(\mathrm{~S}^{4}\right)$ such that $E x=E\left(\beta \eta_{n+1}\right)+2 y$, that is, $E\left(x-\beta \eta_{n+1}\right)=2 y$. We have $\quad x-\beta \eta_{n+1}=\varphi_{n+2} E\left(x-\beta \eta_{n+1}\right)=2 \varphi_{n+2}(y) \in$ $2 \pi_{n+2}\left(\mathrm{~S}^{3}\right)$ by Lemma $4.1(1)$ and so $q_{n}^{*}\left(x-\beta \eta_{n+1}\right)=0$ by (4.1). Therefore $q_{n}^{*}(x)=q_{n}^{*}\left(\beta \eta_{n+1}\right)$. Thus $2 \bar{\beta}=q_{n}^{*}\left(\beta \eta_{n+1}\right)$. This completes the proof.

4.1. $\Gamma_{n}$ for $n=0,1,2,7,8,9$. By (4.3) and Lemma 4.1(2), we obtain the results.

4.2. $\Gamma_{3}$. By (4.3) and Lemma 4.1(2), we have the following exact sequence:

$$
0 \rightarrow \mathbf{Z}_{2}\left\{q_{3}^{*}\left(\eta_{3}^{2}\right)\right\} \stackrel{\subsetneq}{\rightarrow} \Gamma_{3} \stackrel{i_{3}^{*}}{\rightarrow} \mathbf{Z}_{2}\left\{\eta_{3}\right\} \rightarrow 0 .
$$

By setting $\beta=\eta_{3}$ in Lemma 4.4 , we have

$$
2 \overline{\eta_{3}}=q_{3}^{*}\left(\eta_{3}^{2}\right) .
$$

Hence we have the result by Lemma 4.2(2). From now on, we will denote $E^{n} \overline{\eta_{3}}$ by $\overline{\eta_{n+3}}$. 
4.3. $\Gamma_{n}$ for $n=4,15,16$. By (4.3) and Lemma 4.1(2), we have the following exact sequence:

$$
0 \rightarrow \mathbf{Z}_{2}\left\{q_{4}^{*} v^{\prime}\right\} \stackrel{\subset}{\rightarrow} \Gamma_{4} \stackrel{i_{4}^{*}}{\rightarrow} \mathbf{Z}_{2}\left\{\eta_{3}^{2}\right\} \rightarrow 0 .
$$

Since $i_{4}^{*}\left(\eta_{3} \overline{\eta_{4}}\right)=\eta_{3}^{2}$ and $2\left(\eta_{3} \overline{\eta_{4}}\right)=\left(2 \eta_{3}\right) \overline{\eta_{4}}=0$, we obtain the result for $n=4$ by Lemma 4.2(1). By similar reason, we obtain the results for $n=15,16$.

4.4. $\Gamma_{5}$. By (4.3) and Lemma 4.1(2), we have the following exact sequence:

$$
0 \rightarrow \mathbf{Z}_{2}\left\{q_{5}^{*}\left(v^{\prime} \eta_{6}\right)\right\} \stackrel{\ulcorner}{\rightarrow} \Gamma_{5} \stackrel{i_{5}^{*}}{\rightarrow} \mathbf{Z}_{2}\left\{2 v^{\prime}\right\} \rightarrow 0 .
$$

Since $i_{5}^{*}\left(\eta_{3}^{2} \overline{\eta_{5}}\right)=\eta_{3}^{3}=2 v^{\prime}$ by Lemma $4.3(1)$ and $2\left(\eta_{3}^{2} \overline{\eta_{5}}\right)=\left(2 \eta_{3}^{2}\right) \overline{\eta_{5}}=0$, we have the result by Lemma $4.2(1)$.

4.5. $\Gamma_{6}$. By (4.3) and Lemma 4.1(2), we have the following short exact sequence:

$$
0 \rightarrow \mathbf{Z}_{2}\left\{q_{6}^{*}\left(v^{\prime} \eta_{6}^{2}\right)\right\} \stackrel{\ulcorner}{\rightarrow} \Gamma_{6} \stackrel{i_{6}^{*}}{\rightarrow} \mathbf{Z}_{2}\left\{v^{\prime} \eta_{6}\right\} \rightarrow 0 .
$$

Since $i_{6}^{*}\left(v^{\prime} \bar{\eta}_{6}\right)=v^{\prime} \eta_{6}$ and $2\left(v^{\prime} \bar{\eta}_{6}\right)=v^{\prime}\left(2 \bar{\eta}_{6}\right)=v^{\prime}\left(\eta_{6}^{2} q_{6}\right)=q_{6}^{*}\left(v^{\prime} \eta_{6}^{2}\right)$ by (4.4), we have the result by Lemma $4.2(2)$.

4.6. $\Gamma_{10}$. By (4.3) and Lemma 4.1(2), we have the following exact sequence:

$$
0 \rightarrow\left(\mathbf{Z}_{2}\right)^{2}\left\{q_{10}^{*} \mu_{3}, q_{10}^{*}\left(\eta_{3} \varepsilon_{4}\right)\right\} \stackrel{\ulcorner}{\rightarrow} \Gamma_{10} \stackrel{i_{10}^{*}}{\rightarrow} \mathbf{Z}_{2}\left\{\varepsilon_{3}\right\} \rightarrow 0 .
$$

We have $2 \overline{\varepsilon_{3}}=q_{10}^{*}\left(\varepsilon_{3} \eta_{11}\right)=q_{10}^{*}\left(\eta_{3} \varepsilon_{4}\right)$ by Lemma 4.4 and Lemma $4.3(1)$. Hence we obtain the result by Lemma 4.2(2). From now on, we will denote $E^{n} \overline{\varepsilon_{3}}$ by $\overline{\varepsilon_{n+3}}$.

4.7. $\Gamma_{11}$. By (4.3) and Lemma 4.1(2), we have the following exact sequence:

$$
0 \rightarrow\left(\mathbf{Z}_{2}\right)^{2}\left\{q_{11}^{*} \varepsilon^{\prime}, q_{11}^{*}\left(\eta_{3} \mu_{4}\right)\right\} \stackrel{\ulcorner}{\rightarrow} \Gamma_{11} \stackrel{i_{11}^{*}}{\rightarrow}\left(\mathbf{Z}_{2}\right)^{2}\left\{\mu_{3}, \eta_{3} \varepsilon_{4}\right\} \rightarrow 0 .
$$

We have

$$
2 \overline{\mu_{3}}=q_{11}^{*}\left(\mu_{3} \eta_{12}\right)=q_{11}^{*}\left(\eta_{3} \mu_{4}\right)
$$

by Lemma 4.4 and Lemma 4.3(2). On the other hand, $i_{11}^{*}\left(\eta_{3} \overline{\varepsilon_{4}}\right)=\eta_{3} \varepsilon_{4}$ and $2\left(\eta_{3} \bar{\varepsilon}_{4}\right)=\left(2 \eta_{3}\right) \bar{\varepsilon}_{4}=0$. Hence we obtain the result by Lemma 4.2(2). From now on, we will denote $E^{n} \overline{\mu_{3}}$ by $\overline{\mu_{n+3}}$.

4.8. $\Gamma_{12}$. By (4.3) and Lemma 4.1(2), we have the following exact sequence:

$$
0 \rightarrow\left(\mathbf{Z}_{2}\right)^{3}\left\{q_{12}^{*} \mu^{\prime}, q_{12}^{*}\left(\varepsilon_{3} v_{11}\right), q_{12}^{*}\left(v^{\prime} \varepsilon_{6}\right)\right\} \stackrel{\subsetneq}{\rightarrow} \Gamma_{12} \stackrel{i_{12}^{*}}{\rightarrow}\left(\mathbf{Z}_{2}\right)^{2}\left\{2 \varepsilon^{\prime}, \mu_{3} \eta_{12}\right\} \rightarrow 0 .
$$


We have $i_{12}^{*}\left(\eta_{3}^{2} \overline{\varepsilon_{5}}\right)=\eta_{3}^{2} \varepsilon_{5}=2 \varepsilon^{\prime}$ by Lemma $4.3(1)$ and $2\left(\eta_{3}^{2} \overline{\varepsilon_{5}}\right)=\left(2 \eta_{3}^{2}\right) \overline{\varepsilon_{5}}=0$. We have $i_{12}^{*}\left(\mu_{3} \overline{\eta_{12}}\right)=\mu_{3} \eta_{12}$ and $2\left(\mu_{3} \overline{\eta_{12}}\right)=\left(2 \mu_{3}\right) \overline{\eta_{12}}=0$. Hence we obtain the result by Lemma $4.2(1)$.

4.9. $\Gamma_{13}$. By (4.3) and Lemma 4.1(2), we have the following exact sequence:

$$
0 \rightarrow\left(\mathbf{Z}_{2}\right)^{2}\left\{q_{13}^{*}\left(v^{\prime} \mu_{6}\right), q_{13}^{*}\left(v^{\prime} \eta_{6} \varepsilon_{7}\right)\right\} \stackrel{\ulcorner}{\rightarrow} \Gamma_{13} \stackrel{i_{13}^{*}}{\rightarrow}\left(\mathbf{Z}_{2}\right)^{3}\left\{2 \mu^{\prime}, \varepsilon_{3} v_{11}, v^{\prime} \varepsilon_{6}\right\} \rightarrow 0 .
$$

We have $i_{13}^{*}\left(\mu_{3} \eta_{12} \overline{\eta_{13}}\right)=\mu_{3} \eta_{12}^{2}=2 \mu^{\prime}$ by Lemma 4.3 , and $2\left(\mu_{3} \eta_{12} \overline{\eta_{13}}\right)=$ $\left(2 \mu_{3}\right) \eta_{12} \overline{\eta_{13}}=0$. By setting $\beta=\varepsilon_{3} v_{11}, v^{\prime} \varepsilon_{6}$ in Lemma 4.4, we have $2 \overline{\varepsilon_{3} v_{11}}=$ $q_{13}^{*}\left(\varepsilon_{3} v_{11} \eta_{14}\right)=0$ and $2\left(v^{\prime} \overline{\varepsilon_{6}}\right)=q_{13}^{*}\left(v^{\prime} \varepsilon_{6} \eta_{14}\right)=q_{13}^{*}\left(v^{\prime} \eta_{6} \varepsilon_{7}\right)$ from Lemma 4.3(1). Hence we obtain the result by Lemma 4.2(2).

4.10. $\Gamma_{14}$. By (4.3) and Lemma 4.1(2), we have the following exact sequence:

$$
0 \rightarrow \mathbf{Z}_{2}\left\{q_{14}^{*}\left(v^{\prime} \eta_{6} \mu_{7}\right)\right\} \stackrel{ᄃ}{\rightarrow} \Gamma_{14} \stackrel{i_{14}^{*}}{\rightarrow}\left(\mathbf{Z}_{2}\right)^{2}\left\{v^{\prime} \mu_{6}, v^{\prime} \eta_{6} \varepsilon_{7}\right\} \rightarrow 0 .
$$

We have $i_{14}^{*}\left(v^{\prime} \overline{\mu_{6}}\right)=v^{\prime} \mu_{6}$ and $2\left(v^{\prime} \overline{\mu_{6}}\right)=v^{\prime}\left(2 \overline{\mu_{6}}\right)=v^{\prime}\left(\eta_{6} \mu_{7} q_{14}\right)=q_{14}^{*}\left(v^{\prime} \eta_{6} \mu_{7}\right)$ by (4.5). We have $i_{14}^{*}\left(v^{\prime} \eta_{6} \overline{\varepsilon_{7}}\right)=v^{\prime} \eta_{6} \varepsilon_{7}$ and $2\left(v^{\prime} \eta_{6} \overline{\varepsilon_{7}}\right)=v^{\prime}\left(2 \eta_{6}\right) \overline{\varepsilon_{7}}=0$. Hence we obtain the result by Lemma $4.2(2)$.

4.11. $\Gamma_{17}$. By (4.3) and Lemma 4.1(2), we have the following exact sequence:

$$
0 \rightarrow\left(\mathbf{Z}_{2}\right)^{2}\left\{q_{17}^{*}\left(\mu_{3} \sigma_{12}\right), q_{17}^{*}\left(\eta_{3} \bar{\varepsilon}_{4}\right)\right\} \stackrel{\subsetneq}{\rightarrow} \Gamma_{17} \stackrel{i_{17}^{*}}{\rightarrow} \mathbf{Z}_{2}\left\{\bar{\varepsilon}_{3}\right\} \rightarrow 0 .
$$

Since $2 \overline{\bar{\varepsilon}}_{3}=q_{17}^{*}\left(\bar{\varepsilon}_{3} \eta_{18}\right)=q_{17}^{*}\left(\eta_{3} \bar{\varepsilon}_{4}\right)$ by Lemma 4.4 and Lemma $4.3(1)$, we obtain the result by Lemma 4.2(2). From now on, we will denote $E^{n} \overline{\bar{\varepsilon}}_{3}$ by $\overline{\bar{\varepsilon}_{n+3}}$.

4.12. $\Gamma_{18}$. By (4.3) and Lemma 4.1(2), we have the following exact sequence:

$$
0 \rightarrow\left(\mathbf{Z}_{2}\right)^{3}\left\{q_{18}^{*} \bar{\varepsilon}^{\prime}, q_{18}^{*} \bar{\mu}_{3}, q_{18}^{*}\left(\eta_{3} \mu_{4} \sigma_{13}\right)\right\} \stackrel{\subsetneq}{\rightarrow} \Gamma_{18} \stackrel{i_{18}^{*}}{\rightarrow}\left(\mathbf{Z}_{2}\right)^{2}\left\{\mu_{3} \sigma_{12}, \eta_{3} \bar{\varepsilon}_{4}\right\} \rightarrow 0 .
$$

We have $2 \overline{\mu_{3} \sigma_{12}}=q_{18}^{*}\left(\mu_{3} \sigma_{12} \eta_{19}\right)=q_{18}^{*}\left(\eta_{3} \mu_{4} \sigma_{13}\right)$ by Lemma 4.4 and Lemma 4.3. We have $i_{18}^{*}\left(\bar{\varepsilon}_{3} \bar{\eta}_{18}\right)=\bar{\varepsilon}_{3} \eta_{18}=\eta_{3} \bar{\varepsilon}_{4}$ by Lemma $4.3(1)$ and $2\left(\bar{\varepsilon}_{3} \overline{\eta_{18}}\right)=\left(2 \bar{\varepsilon}_{3}\right) \overline{\eta_{18}}=0$. Hence we obtain the result by Lemma 4.2(2). From now on, we will denote $E^{n} \overline{\mu_{3} \sigma_{12}}$ by $\overline{\mu_{n+3} \sigma_{n+12}}$.

4.13. $\Gamma_{19}$. By (4.3) and Lemma 4.1(2), we have the following exact sequence:

$$
0 \rightarrow\left(\mathbf{Z}_{2}\right)^{3}\left\{q_{19}^{*}\left(\mu^{\prime} \sigma_{14}\right), q_{19}^{*}\left(v^{\prime} \bar{\varepsilon}_{6}\right), q_{19}^{*}\left(\eta_{3} \bar{\mu}_{4}\right)\right\} \stackrel{ᄃ}{\rightarrow} \Gamma_{19} \stackrel{i_{19}^{*}}{\rightarrow}\left(\mathbf{Z}_{2}\right)^{3}\left\{2 \bar{\varepsilon}^{\prime}, \bar{\mu}_{3}, \eta_{3} \mu_{4} \sigma_{13}\right\} \rightarrow 0 .
$$

We have $i_{19}^{*}\left(\bar{\varepsilon}_{3} \eta_{18} \bar{\eta}_{19}\right)=\bar{\varepsilon}_{3} \eta_{18}^{2}=2 \bar{\varepsilon}^{\prime}$ by Lemma 4.3 and $2\left(\bar{\varepsilon}_{3} \eta_{18} \overline{\eta_{19}}\right)=$ $\left(2 \bar{\varepsilon}_{3}\right) \eta_{18} \bar{\eta}_{19}=0$. We have $2 \bar{\mu}_{3}=q_{19}^{*}\left(\bar{\mu}_{3} \eta_{20}\right)=q_{19}^{*}\left(\eta_{3} \bar{\mu}_{4}\right)$ by Lemma 4.4 and Lemma $4.3(2)$. We have $i_{19}^{*}\left(\mu_{3} \sigma_{12} \overline{\eta_{19}}\right)=\mu_{3} \sigma_{12} \eta_{19}=\eta_{3} \mu_{4} \sigma_{13}$ by Lemma 4.3 
and $2\left(\mu_{3} \sigma_{12} \overline{\eta_{19}}\right)=\left(2 \mu_{3}\right) \sigma_{12} \overline{\eta_{19}}=0$. Hence we obtain the result by Lemma 4.2(2). From now on, we will denote $E^{n} \overline{\bar{\mu}}_{3}$ by $\overline{\bar{\mu}_{n+3}}$.

4.14. $\Gamma_{20}$. By (4.3) and Lemma 4.1(2), we have the following exact sequence:

$$
0 \rightarrow\left(\mathbf{Z}_{2}\right)^{2}\left\{q_{20}^{*}\left(\bar{\mu}^{\prime}\right), q_{20}^{*}\left(v^{\prime} \mu_{6} \sigma_{15}\right)\right\} \stackrel{ᄃ}{\rightarrow} \Gamma_{20} \stackrel{i_{20}^{*}}{\rightarrow}\left(\mathbf{Z}_{2}\right)^{3}\left\{2\left(\mu^{\prime} \sigma_{14}\right), v^{\prime} \bar{\varepsilon}_{6}, \eta_{3} \bar{\mu}_{4}\right\} \rightarrow 0 .
$$

We have $i_{20}^{*}\left(\mu_{3} \sigma_{12} \eta_{19} \overline{\eta_{20}}\right)=\mu_{3} \sigma_{12} \eta_{19}^{2}=2\left(\mu^{\prime} \sigma_{14}\right)$ by Lemma 4.3 and $2\left(\mu_{3} \sigma_{12} \eta_{19} \overline{\eta_{20}}\right)$ $=\left(2 \mu_{3}\right) \sigma_{12} \eta_{19} \bar{\eta}_{20}=0$. We have $i_{20}^{*}\left(v^{\prime} \overline{\bar{\varepsilon}}_{6}\right)=v^{\prime} \bar{\varepsilon}_{6}$ and $i_{20}^{*}\left(\eta_{3} \overline{\bar{\mu}}_{4}\right)=\eta_{3} \bar{\mu}_{4}$. It follows from Lemma 4.3 and Lemma 4.4 that $2\left(v^{\prime} \overline{\bar{\varepsilon}}_{6}\right)=q_{20}^{*}\left(v^{\prime} \bar{\varepsilon}_{6} \eta_{21}\right)=q_{20}^{*}\left(\bar{\varepsilon}_{3} v_{18} \eta_{21}\right)=0$ and $2\left(\eta_{3} \bar{\mu}_{4}\right)=q_{20}^{*}\left(\eta_{3} \bar{\mu}_{4} \eta_{21}\right)=q_{20}^{*}\left(\eta_{3}^{2} \bar{\mu}_{5}\right)=q_{20}^{*}\left(2 \bar{\mu}^{\prime}\right)=0$. Therefore we obtain the result by Lemma $4.2(1)$.

4.15. $\Gamma_{21}$. By (4.3) and Lemma 4.1(2), we have the following exact sequence:

$$
0 \rightarrow\left(\mathbf{Z}_{2}\right)^{2}\left\{q_{21}^{*}\left(v^{\prime} \bar{\mu}_{6}\right), q_{21}^{*}\left(v^{\prime} \eta_{6} \mu_{7} \sigma_{16}\right)\right\} \stackrel{\subsetneq}{\rightarrow} \Gamma_{21} \stackrel{i_{21}^{*}}{\rightarrow}\left(\mathbf{Z}_{2}\right)^{2}\left\{2 \bar{\mu}^{\prime}, v^{\prime} \mu_{6} \sigma_{15}\right\} \rightarrow 0 .
$$

We have $i_{21}^{*}\left(\bar{\mu}_{3} \eta_{20} \overline{\eta_{21}}\right)=\bar{\mu}_{3} \eta_{20}^{2}=2 \bar{\mu}^{\prime}$ by Lemma 4.3 and $2\left(\bar{\mu}_{3} \eta_{20} \overline{\eta_{21}}\right)=$ $\left(2 \bar{\mu}_{3}\right) \eta_{20} \overline{\eta_{21}}=0$. We have $i_{21}^{*}\left(v^{\prime} \overline{\mu_{6} \sigma_{15}}\right)=v^{\prime} \mu_{6} \sigma_{15}$ and $2\left(v^{\prime} \overline{\mu_{6} \sigma_{15}}\right)=q_{21}^{*}\left(v^{\prime} \mu_{6} \sigma_{15} \eta_{22}\right)$ $=q_{21}^{*}\left(v^{\prime} \eta_{6} \mu_{7} \sigma_{16}\right)$ by Lemmas 4.4 and 4.3. Hence we obtain the result by Lemma 4.2(2). This completes the proof of Theorem 2.2.

\section{Proof of Proposition 2.4}

Let $\operatorname{map}\left(\mathbf{P}^{3}, \mathbf{P}^{3}\right)$ denote the space of self maps of $\mathbf{P}^{3}$ not necessarily preserving base point. This is a monoid with respect to the composition operation. Borsuk's fibre theorem [5, Proposition (6.34)] says that the evaluation map $e v: \operatorname{map}\left(\mathbf{P}^{3}, \mathbf{P}^{3}\right) \rightarrow \mathbf{P}^{3}, f \mapsto f(*)$, is a fibration whose fibre is $\operatorname{map}_{*}\left(\mathbf{P}^{3}, \mathbf{P}^{3}\right)$, where the base point $*$ is the unit of the group $\mathbf{P}^{3}$. By [12, Theorems IIa, IIb], we have

$$
\begin{aligned}
\pi_{0}\left(\operatorname{map}\left(\mathbf{P}^{3}, \mathbf{P}^{3}\right)\right) & =\pi_{0}\left(\operatorname{map}_{*}\left(\mathbf{P}^{3}, \mathbf{P}^{3}\right)\right) \\
& =\left[\mathbf{P}^{3}, \mathbf{P}^{3}\right] \underset{\cong}{\stackrel{\xi}{\longrightarrow}} \operatorname{Hom}\left(H^{3}\left(\mathbf{P}^{3} ; \mathbf{Z}\right), H^{3}\left(\mathbf{P}^{3} ; \mathbf{Z}\right)\right) \cong \mathbf{Z}
\end{aligned}
$$

where $\xi$ assigns $f^{*}$ to the homotopy class of $f \in \operatorname{map}_{*}\left(\mathbf{P}^{3}, \mathbf{P}^{3}\right)$. Hence $\operatorname{aut}\left(\mathbf{P}^{3}\right)$ and aut $_{*}\left(\mathbf{P}^{3}\right)$ consist of two path components of $\operatorname{map}\left(\mathbf{P}^{3}, \mathbf{P}^{3}\right)$ and $\operatorname{map}_{*}\left(\mathbf{P}^{3}, \mathbf{P}^{3}\right)$, respectively. Therefore $\pi_{0}\left(\operatorname{aut}\left(\mathbf{P}^{3}\right)\right) \cong \pi_{0}\left(\operatorname{aut}_{*}\left(\mathbf{P}^{3}\right)\right) \cong \mathbf{Z}_{2}$ and $\pi_{n}\left(\operatorname{aut}\left(\mathbf{P}^{3}\right), 1_{\mathbf{P}^{3}}\right) \cong$ $\pi_{n}\left(\operatorname{map}\left(\mathbf{P}^{3}, \mathbf{P}^{3}\right), 1_{\mathbf{P}^{3}}\right)$ and $\pi_{n}\left(\operatorname{aut}_{*}\left(\mathbf{P}^{3}\right), 1_{\mathbf{P}^{3}}\right) \cong \pi_{n}\left(\operatorname{map}_{*}\left(\mathbf{P}^{3}, \mathbf{P}^{3}\right), 1_{\mathbf{P}^{3}}\right)$ for $n \geq 1$. For any $x \in \mathbf{P}^{3}$, let $L_{x}: \mathbf{P}^{3} \rightarrow \mathbf{P}^{3}$ denote the map $y \mapsto x y$. Then the map $\mathbf{P}^{3} \rightarrow \operatorname{map}\left(\mathbf{P}^{3}, \mathbf{P}^{3}\right), \quad x \mapsto L_{x}$, is a cross section of $e v$. Hence the homotopy exact sequence of the fibration $e v$ splits so that $\pi_{n}\left(\operatorname{map}\left(\mathbf{P}^{3}, \mathbf{P}^{3}\right), 1_{\mathbf{P}^{3}}\right) \cong$ $\pi_{n}\left(\operatorname{map}_{*}\left(\mathbf{P}^{3}, \mathbf{P}^{3}\right), 1_{\mathbf{P}^{3}}\right) \oplus \pi_{n}\left(\mathbf{P}^{3}\right)$. Since all path-components of $\operatorname{map}\left(\mathbf{P}^{3}, \mathbf{P}^{3}\right)$ have the same homotopy type, $\pi_{n}\left(\operatorname{map}\left(\mathbf{P}^{3}, \mathbf{P}^{3}\right), f\right) \cong \pi_{n}\left(\operatorname{map}\left(\mathbf{P}^{3}, \mathbf{P}^{3}\right), 0\right)$ for every 
$f \in \operatorname{map}\left(\mathbf{P}^{3}, \mathbf{P}^{3}\right)$. Similarly all path components of the following spaces have the same homotopy type, respectively: $\operatorname{map}_{*}\left(\mathbf{P}^{3}, \mathbf{P}^{3}\right)$, aut $\left(\mathbf{P}^{3}\right)$ and $\operatorname{aut}_{*}\left(\mathbf{P}^{3}\right)$. Therefore if $n \geq 1$, then we have $\pi_{n}\left(\operatorname{aut}_{*}\left(\mathbf{P}^{3}\right)\right) \cong \pi_{n}\left(\operatorname{map}_{*}\left(\mathbf{P}^{3}, \mathbf{P}^{3}\right)\right)$ and $\pi_{n}\left(\operatorname{aut}\left(\mathbf{P}^{3}\right)\right)$ $\cong \pi_{n}\left(\operatorname{map}\left(\mathbf{P}^{3}, \mathbf{P}^{3}\right)\right) \cong \pi_{n}\left(\operatorname{map}_{*}\left(\mathbf{P}^{3}, \mathbf{P}^{3}\right)\right) \oplus \pi_{n}\left(\mathbf{P}^{3}\right)$. Hence we obtain Proposition 2.4 by Proposition 2.1.

\section{REFERENCES}

[ 1 ] J. C. Becker and D. H. Gottlieb, Coverings of fibrations, Compositio Mathematica 26 (1973), 119-128.

[2] W. Browder and E. Spanier, $H$-spaces and duality, Pacific J. Math. 12 (1970), 411-414.

[3] T. Egawa and H. Ōshima, Maps between small Hopf spaces, Math. J. Ibaraki Univ. 32 (2000), 33-61.

[4] I. M. JAmes, On sphere-bundles over spheres, Comment. Math. Helv. 35 (1961), 126-135.

[5] I. M. JAMES, General topology and homotopy theory, Springer-Verlag, Berlin, 1984.

[6] A. Kono AND H. Ōshima, Commutativity of the group of self-homotopy classes of Lie groups, Bull. London Math. Soc. 36 (2004), 37-52.

[7] K. Maruyama and H. Ōshima, Homotopy groups of the space of self-maps of Lie groups, J. Math. Soc. Japan 60 (2008), 767-792.

[ 8 ] M. Mimura and H. Ōshima, Self homotopy groups of Hopf spaces with at most three cells, J. Math. Soc. Japan 51 (1999), 71-92.

[9] M. Mimura and H. Toda, The $n+20$-th homotopy groups of $n$-spheres, J. Math. Kyoto Univ. 3 (1963), 37-58.

[10] M. Mimura and H. Toda, Homotopy groups of $\mathrm{SU}(3), \mathrm{SU}(4)$ and $\mathrm{Sp}(2)$, J. Math. Kyoto Univ. 3 (1964), 217-250

[11] K. ÔGUCHI, Generators of 2-primary components of homotopy groups of spheres, unitary groups and symplectic groups, J. Fac. Sci. Univ. Tokyo 11 (1964), 65-111.

[12] P. Olum, Mappings of manifolds and the notion of degree, Ann. of Math. 58 (1953), 458-480.

[13] H. Ōshima, Self homotopy set of a Hopf space, Quart. J. Math. 50 (1999), 483-495.

[14] H. Ōshima, Self homotopy group of the exceptional Lie group $G_{2}$, J. Math. Kyoto Univ. 40 (2000), 177-184.

[15] H. Ōshima, The group of self homotopy classes of SO(4), J. Pure App. Algebra 185 (2003), 193-205.

[16] K. Ōshima AND H. Ōshima, Homotopy groups of the spaces of self-maps of Lie groups II, Kodai Math. J. 32 (2009), 530-546.

[17] E. ReEs, Multiplications on projective spaces, Michigan Math. J. 16 (1969), 297-302.

[18] H. Toda, Composition methods in homotopy groups of spheres, Ann. of math. studies 49, Princeton, 1962.

Katsumi Ōshima

IBARAKI UNIVERSITY

Mito, Ibaraki 310-8512

JAPAN

E-mail: oshimakatsumi@hotmail.co.jp 